\title{
Deep Sequencing from henl Mutants to Identify Small RNA 3' Modifications
}

\author{
J. Zhai AND B.C. Meyers \\ Department of Plant \& Soil Sciences, and Delaware Biotechnology Institute, \\ University of Delaware, Newark, Delaware 19711 \\ Correspondence: meyers@dbi.udel.edu
}

\begin{abstract}
microRNAs (miRNAs) function via targeting of messenger RNAs, suppressing protein levels, and playing important roles in biological processes of plants and animals. The pathway for miRNA biogenesis is well established, but less is known about miRNA turnover, largely because of difficulties in capturing miRNAs during the process of decay, in which they are both rare and ephemeral. The HEN1 protein methylates the $3^{\prime}$ terminus of small RNAs (sRNAs), protecting them from poly-urydilation and degradation. Recent progress using deep sequencing to study sRNAs in henl reveals the potential for henl mutants to serve as a platform for studies of miRNA turnover, with the sequencing data providing unprecedented precision and detail in the characterization of $3^{\prime}$ modifications.
\end{abstract}

In plants, microRNAs (miRNAs) (Reinhart et al. 2002; Jones-Rhoades et al. 2006) and small interfering RNAs (siRNAs) (Hamilton and Baulcombe 1999; Chapman and Carrington 2007) are the two major classes of sRNAs. microRNAs in plants function in a wide variety of roles principally in development but have demonstrated roles in modulation of transcriptional responses to abiotic and biotic stress, and to nutrient deficiencies (Chen 2005; Sunkar et al. 2007; Rubio-Somoza et al. 2009; Jeong et al. 2011). siRNAs in plants are predominantly but not exclusively composed of heterochromatic siRNAs that function in genome defense via the maintenance of heterochromatin, presumably important for the suppression of transposon activity (Volpe and Martienssen 2011).

miRNAs are processed from long single-stranded transcripts that originate from distinct genomic loci and are transcribed by RNA polymerase II (Pol II) like a typical messenger RNA (mRNA). These miRNA precursor transcripts fold into imperfectly paired, hairpin-like structures and are subsequently processed via a series of nuclearlocalized events (in plants, because some processing events in animals occur in the cytoplasm) that lead to the generation of a single, mature sRNA of typically 21 or 22 nucleotides (Bartel 2004; Voinnet 2009). In an early processing step, an sRNA "duplex" comprised of both the miRNA and miRNA-star is cleaved from the precursor hairpin by the DICER-LIKE1 (DCL1) protein. The miRNA strand, also known as the "guide" molecule eventually becomes associated with (or "loaded" into) an ARGONAUTE (AGO) protein to form an active RNA-induced silencing complex (RISC). The miRNA-star strand is usually nonfunctional (hence, it is also known as the "passenger" strand), and it is preferentially decayed leading to a substantially lower abundance than the miRNA. In contrast, siRNAs are often not per se encoded in the genome but are derived from precursor RNA molecules that have been made double stranded by RNA-dependent RNA polymerases (RDRs). These double-stranded RNA (dsRNA) precursors are processed by DCL proteins into multiple distinct siRNA classes that represent both strands of the original dsRNA (Baulcombe 2004; Chapman and Carrington 2007). Plant siRNAs come in several forms; for example, heterochromatic siRNAs are produced from RNA polymerase IVdependent precursors generated from a wide variety of repetitive sequences (Wierzbicki et al. 2008), whereas trans-acting or phased siRNAs are produced via Pol II from long, noncoding mRNAs or even from protein-coding transcripts (Montgomery et al. 2008; Cuperus et al. 2010; Zhai et al. 2011). The Arabidopsis genome includes four $D C L$ genes that are responsible for generating distinct classes of sRNAs and of different sizes, ranging from 21 to 24 nucleotides. Mutant analysis has revealed some functional redundancies among the $D C L$ genes (Deleris et al. 2006; Henderson et al. 2006). Despite the diversification of sRNA types and biogenesis pathway proteins, current data suggest that all plant sRNAs require $3^{\prime}$ methylation performed by a protein known as HEN1. This protein also has a conserved function in the maturation of specific subclasses (namely, the Piwi-interacting RNAs [piRNAs]) of animal sRNAs. Deep sequencing of sRNAs in mutants of hen 1 can describe the types of $3^{\prime}$ modifications to which sRNAs are subjected in the absence of a protective methyl group. In this chapter, we discuss the use of these deep sequencing data, types of modifications that are observed, and insights that may be made from these analyses when performed on hen 1 mutants.

\section{Discovery of HEN1 and Its Conserved Function in Many Species}

The HEN1 (HUA ENHANCER1) gene was first identified in a screen for Arabidopsis floral development mu- 
tants (Chen et al. 2002). The phenotype was identified as an enhancer of a floral-organ phenotype in the hual-1 hua2-1 double mutant; when the Chen laboratory segregated out the hen 1 mutant from the hua mutant genes, the single-gene mutant henl was observed to have a pleiotropic phenotype, including a short stature, delayed flowering, reduced fertility, and multiple inflorescence aberrations (Chen et al. 2002). These phenotypes were later revealed to be attributable to widespread reductions in sRNAs and their activities. In subsequent work by the Chen laboratory, the HEN1 protein was shown to function as a methyltransferase; the methyltransferase activity is specific to sRNAs, adding a $2^{\prime}$-O-methyl group on the $3^{\prime}$-terminal nucleotide of template sRNAs (Yu et al. 2005; Yang et al. 2006). In more recent work, the crystal structure of HEN1 suggests that it can specifically recognize the sRNA duplex as its template via multiple RNA-binding domains and a methyltransferase domain (Huang et al. 2009). Thus, HEN1 activity is highly specific, but a loss of HEN1 can have a widespread impact on sRNAs, particularly in plants in which all known small RNA pathways converge on the methylation activity of HEN1.

miRNAs examined by RNA gel blot analysis show unusual length heterogeneity in a henl mutant background ( $\mathrm{Li}$ et al. 2005). Comparisons performed by cloning and sequencing individual miRNAs in wild-type and hen 1-1 backgrounds showed the addition of predominantly uridine (U) nucleotides at the $3^{\prime}$ end of some miRNAs in a hen 1-1 mutant ( $\mathrm{Li}$ et al. 2005). This U "tailing" is presumably a result of deprotection caused by a lack of $3^{\prime}$ methylation owing to the loss of HEN1 activity. RNA gel blot analysis of miRNAs has shown that miRNAs in plant hen 1 mutants display a "laddering" of length, reflecting the $3^{\prime}$ tails, whereas sizes shorter than the wild-type length are also observed, suggesting that $3^{\prime}$ truncation occurs as well (Fig. 1A) (Li et al. 2005). As we describe below, our analyses of deep sequencing data have confirmed that both $3^{\prime}$ truncation and $3^{\prime}$ tailing are common for most miRNAs in plant hen 1 mutants. An additional and likely related observation was that the abundance of most miRNAs is considerably reduced in the hen $1 \mathrm{mu}-$ tants, with no corresponding decrease in the rate of miRNA biogenesis, implying that in hen 1, miRNAs have increased rates of degradation ( $\mathrm{Li}$ et al. 2005; Yu et al. 2005). At that point in time, it was unclear whether $\mathrm{U}$ tailing is a cause or a consequence of miRNA degradation. The $3^{\prime}$ tailing of miRNAs is easily detected by today's "next-generation" sequencing, and thus, sRNAs in plant hen 1 mutant are good candidates for molecular characterization using modern techniques.

The hen 1-1 allele in the Arabidopsis Landsberg erecta (Ler) ecotype came out of an enhancer screen of the hual-1/hua2-1 background (Chen et al. 2002). It was the first hen 1 mutant to be identified in any species and therefore contributed the name by which even animal HEN1 genes are now known. In Arabidopsis, a variety of alleles have since been characterized via both forwardand reverse-genetics approaches. These include the weaker hen 1-2 allele, also from Ler, and the identical point mutation in the Columbia ( $\mathrm{Col}$ ) background isolated from an independent genetic screen and known as hen 1-8 (Yu et al. 2010); curiously, the same allele in different backgrounds produces different phenotypes, with a more severe developmental impact (on fertility, for example) in the Col background as well as greater molecular effects such as a reduction in miRNA levels (Yu et al. 2010). In rice, two mutant alleles in the ortholog WAVY LEAF1 (WAF1) of Arabidopsis HEN1 have been described; the rice wafl-1 and wafl-2 mutants each bear a single-base substitution, leading to a premature stop codon in the second exon and a nonfunctional splicing site of the fourth intron, respectively (Abe et al. 2010). Whereas HEN1 is also single copy in rice, there are numerous phenotypic differences in the rice hen1 ("waf") mutants, compared with Arabidopsis, most of which reflect a more severe impact on normal biological functions. For example, rice hen 1 mutants show seedling lethality and phenotypes reminiscent of trans-acting siRNA (tasiRNA)-defective mutants in rice (Abe et al. 2010). Because mutants in the rice ortholog were identified only recently, work on the plant HEN1 gene has been primarily performed in Arabidopsis; however, the characterization of wavy leafl (waf1) mutants represents an opportunity to expand our understanding of HEN1 function in monocots.

Besides plants, HEN1 orthologs have also been described and studied in metazoans, in which HEN1 methylates different subsets of sRNAs, primarily including piRNAs but excluding miRNAs (see Table 1 for a summary). piRNAs are a class of animal sRNAs that are abundant in germline cells; they are longer and distinct from miRNAs and target transposons to effect silencing (Juliano et al. 2011). Unlike animal miRNAs that are unmethylated (as a reminder, plant miRNAs are methylated), piRNAs are methylated, a modification performed by HEN1. Thus, the mouse HEN1 homolog ( $m H E N 1)$ is expressed specifically in the testes, in which it functions to methylate piRNAs (Kirino and Mourelatos 2007a,b; Ohara et al. 2007). HEN1 substrates in Drosophila are determined by AGO binding: AGO1-bound miRNAs remain unmethylated, but AGO2-bound siRNAs and Piwi-bound piRNAs are methylated (Horwich et al. 2007; Iwasaki et al. 2009). This suggests that animal HEN1 acts on AGO-bound single-stranded sRNAs (ssRNAs), whereas the plant HEN1 acts on sRNA duplexes before they are incorporated into AGO proteins. The HEN1 homolog in zebrafish has also been studied; as in Drosophila and mouse, the zebrafish HEN1 methylates piRNAs in germline cells and is required for oocyte development, and deep sequencing of sRNAs in the zebrafish hen 1 mutant revealed addition of $U$ tailing on the unmethylated piRNAs (Kamminga et al. 2010). Although miRNAs in animals mostly remain unmethylated, they are fully methylated in plants as a crucial step for stabilization and protection from enzymatic activities such as the $3^{\prime}$ truncation and uridylation mentioned above. miRNAs in Arabidopsis hen 1 mutants, without the protection of $3^{\prime}$ methylation, are often uridylated and in some cases truncated. 

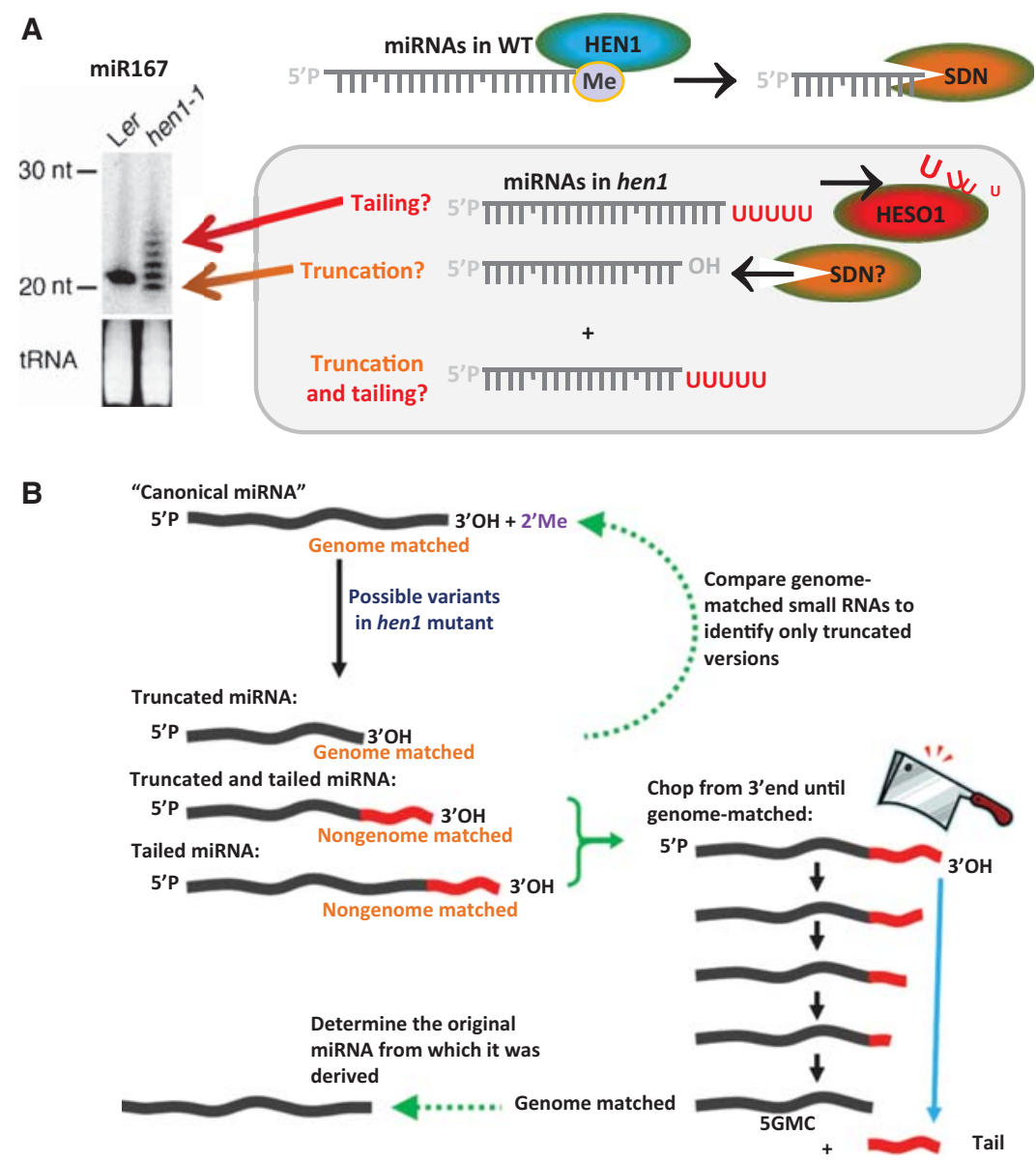

Figure 1. Data handling for truncation and tailing analysis. (A) miRNAs may be $3^{\prime}$ modified in several ways in plant hen 1 mutants. The blot on the left shows the laddering effect observed for miR167 in an Arabidopsis hen 1-1 mutant versus a Landsberg erecta (Ler) control; reprinted from Li et al. (2005). At top right, in wild-type (WT) plants, miRNAs are $3^{\prime}$ methylated by HEN1, but may be decayed by the $3^{\prime}$ to $5^{\prime}$ exonuclease SDN1/2. In the lower box at right, in a hen 1 mutant, miRNAs may be $3^{\prime}$ tailed by HESO1, $3^{\prime}$ truncated by an as-yet-unknown protein (perhaps one of the SDN family of nucleases) or may have some combination of both $3^{\prime}$ truncation and $3^{\prime}$ tailing. (B) Schema for analysis of sRNA truncation and tailing from deep sequencing data, as described in the main text. For sRNA reads that are not genome matched, one nucleotide is chopped off from the $3^{\prime}$ end, in successive rounds until the remaining $5^{\prime}$ sequence perfectly maps to genome. Thus, any nongenome-matched sRNA read could be split into two parts: the longest $5^{\prime}$ genome-matched component (5GMC) and a $3^{\prime}$ "tail." With all reads processed into the format of the 5GMC plus tail, the 5GMC of each read is aligned to annotated miRNAs in the miRNA database (miRBase) for the genome of origin. This then determines the extent of "tailing" (additional of nonconventional 3' nucleotides) and "truncation" (shortening of the miRNA from the 3' end), as diagrammed in $A$. Each miRNA in an organism could have a different, distinguishable pattern of $3^{\prime}$ modifications.

\section{$3^{\prime}$ Modifications of Plant sRNAs: HEN1 and Its Sidekicks}

The Chen laboratory and collaborators have published biochemical data demonstrating the specificity of HEN1 activity. Their initial work in this area showed the methyltransferase activity by which HEN1 transfers a methyl group to the $2^{\prime}$ hydroxyl of the 3 '-terminal nucleotide of a sRNA (Yu et al. 2005; Yang et al. 2006). They showed that both strands of either an miRNA/miRNA* duplex siRNA/siRNA* duplex are methylated in vitro, and HEN1 has a preference for 21-24-nucleotide RNA duplexes with a 2-nucleotide overhang typical of DICER cleavage (Yang et al. 2006). HEN1 in plants possesses double-stranded RNA-binding domains (dsRBDs), domains missing in animal orthologs, and structural data showed that plant HEN1 uses the dsRBDs to recognize $\sim 16$ bps of its duplexed sRNA substrate, measuring the length of the substrate but transferring the methyl group to the duplex in a nonsequence-specific manner (Huang et al. 2009). The transfer of the methyl group to the sRNA duplex occurs via a novel mechanism dependent on $\mathrm{Mg}^{2+}$, coordinated between four invariant residues in the active site of the methyltransferase domain and the $2^{\prime}$ and $3^{\prime}$ hydroxyls on the $3^{\prime}$-terminal nucleotide of the template (Huang et al. 2009). Analogous biochemical work on the mouse HEN1 protein has shown that its activity is specific for single-stranded piRNAs (Kirino and Mourelatos 2007a), consistent with the lack of a dsRBD in the animal orthologs identified to date. Therefore, HEN1 proteins function to add methyl groups to the 
Table 1. HEN1 homologs in various species

\begin{tabular}{|c|c|c|c|c|c|}
\hline Name & Organism & Mutant alleles & Substrates & $\begin{array}{l}\text { sRNAs } \\
\text { sequenced? }\end{array}$ & Reference(s) \\
\hline HEN1 & Arabidopsis & $\begin{array}{l}\text { hen } 1-1, \text { hen } 1-2, \\
\text { hen } 1-4, \text { hen } 1-8\end{array}$ & miRNAs and siRNAs & Yes & Li et al. 2005; Zhao et al. 2012 \\
\hline WAF & Rice & waf -1, waf -2 & miRNAs and siRNAs & No & Abe et al. 2010 \\
\hline mHEN1 & Mouse & $\mathrm{N} / \mathrm{A}$ & piRNAs & No & Kirino and Mourelatos 2007a,b \\
\hline $\begin{array}{l}\text { HEN1 } \\
\text { homolog }\end{array}$ & Zebrafish & henl & piRNAs & Yes & Kamminga et al. 2010 \\
\hline DmHEN1 & Drosophila & hen $1^{f 00810}$ & siRNAs and piRNAs & Yes & $\begin{array}{l}\text { Horwich et al. 2007; Ameres et al. } \\
\text { 2010; Ghildiyal et al. } 2010\end{array}$ \\
\hline Hen $1 p$ & Tetrahymena & $\triangle H E N 1$ & scnRNA & No & Kurth and Mochizuki 2009 \\
\hline
\end{tabular}

$3^{\prime}$ end of small RNAs, with no sequence specificity for the substrate yet shown other than a preference for either ssRNA (in animals) or dsRNA (in plants).

Other than HEN1, several proteins have been identified for their role in modifying the $3^{\prime}$ end of plant sRNAs. These proteins, functioning as "sidekicks" to HEN1 in $3^{\prime}$ modifications, use unmethylated sRNAs as substrates, presumably those either missed by HEN1 or demethylated via natural processes. Work from the Chen laboratory in Arabidopsis has identified a family of SDN (small RNA degrading nuclease) proteins that degrade mature miRNAs (Ramachandran and Chen 2008). Whereas it is still unclear which proteins are responsible for the $3^{\prime}$ truncation that occurs at a higher rate in hen 1 mutants (Fig. 1A), SDN proteins are candidates for this activity. However, in vitro analysis of the SDNs suggests that they degrade miRNAs $3^{\prime}$ to $5^{\prime}$, leaving a small fragment of 8 or 9 nucleotides in length, and this is quite distinct from the typical 1- or 2-nucleotide shorter size observed for truncated miRNAs in RNA gel blots from henl mutants ( $\mathrm{Li}$ et al. 2005; Yu et al. 2005). More recent work from Chen and Yu laboratories identified a nucleotidyl transferase HEN1 SUPPRESSOR1 (HESO1) that is responsible for the addition of the uridine-rich tail to the end of the miRNAs, which are not protected by methylation (Ren et al. 2012; Zhao et al. 2012). Other genes in the same family, which include poly(U) polymerases, were previously shown in animals to modify and regulate the stability of the pre-miRNA (cleaved hairpin) of let-7 (Lehrbach et al. 2009). In the green alga Chlamydomonas, it was shown that uridylation added by nucleotidyltransferase MUT68 can promote the degradation of miRNAs and siRNAs (Ibrahim et al. 2010) and in C. elegans, CDE-1 uridylates 22GRNAs in the germline to promote their degradation and thus prevent over accumulation (van Wolfswinkel et al. 2009). The methylation of sRNAs by HEN1 blocks both $3^{\prime}$ truncation and $3^{\prime}$ tailing, suggesting that either or both of these $3^{\prime}$ modifications may be important marks for RNA destabilization.

\section{Bioinformatics Analysis of sRNAs from hen1 Mutants}

Nearly all studies of $3^{\prime}$ modifications made to miRNAs in henl mutants were performed with RNA gel blots ("northerns") that can only show the changes in miRNA length and relative abundance but cannot identify precisely any changes in miRNA sequence composition. In earlier work, cloning followed by Sanger sequencing of miR173 and miR167 in hen $1-1$ revealed $3^{\prime}$ truncation as well as $1-5$ nucleotides of uridylation at the $3^{\prime}$ end of miRNAs, consistent with observations on the blots (Fig. 1A) (Li et al. 2005). With the tremendous advances in high-throughput sequencing technologies, it is possible to systematically study sRNA populations in hen 1 mutants with unprecedented depth. We have been performing such studies, and although the results are both too extensive to report here and as-yet incomplete, the methodologies that we have devised may have utility for the analysis of mutants of hen 1 and the "sidekick" genes that are also involved in $3^{\prime}$ sRNA modifications.

Our bioinformatics and visualization pipeline assists in the characterization of $3^{\prime}$-end modifications of miRNAs detected using deep sequencing data, typically from an Illumina sequencing instrument. The first step in our process is to identify nongenome-matched sRNA sequences, because most 3' "tailed" sRNAs will no longer match the genome (unless there is a chance identity between the tail and the adjacent nucleotides in the precursor) (Fig. 1B). For sRNA reads that were not genome matched, 1 nucleotide was chopped off from the $3^{\prime}$ end in successive rounds until the remaining $5^{\prime}$ sequence can be mapped to the Arabidopsis genome with no mismatches (Fig. 1B). Thus, any initially nongenome-matched sRNA read could be split into two parts: the longest $5^{\prime}$ genomematched component (5GMC) and a $3^{\prime}$ "tail." The tail is nonexistent for reads that matched the genome perfectly in the first round of analysis. For subsequent analyses, we focus on miRNAs because plant heterochromatic siRNAs are both too variable in sequence (owing to their typical origins from repetitive sequences) and too weakly abundant. With all reads processed into the format of 5GMC plus tail, we can also determine the degree of $3^{\prime}$ truncation relative to annotated, mature miRNAs sequences by comparison of the $5 \mathrm{GMC}$ to all annotated miRNAs in miRBase for the species of interest (Griffiths-Jones et al. 2008). This then determines the extent and composition of the tail (additional, noncanonical $3^{\prime}$ nucleotides) and truncation (shortening of the miRNA from the $3^{\prime}$ end). The sequence data provide the exact composition of the truncated miRNA and the added $3^{\prime}$ tails. 
We have also devised a graphic view that simultaneously displays the degree of both truncation and tailing for specific sRNAs (usually individual miRNAs) (Fig. 2). In this image, the wild-type or canonical miRNA length is assigned to the position in the lower right of a grid. Using the 5GMC and tailing lengths determined as described above, miRNA derivatives of identical length can be assigned to different positions in the grid based on the 5GMC value ( $x$ axis, indicating truncation) and the tail length ( $y$ axis). The size of the spot at each position in the grid corresponds to the proportion of total reads for a given sRNA that had that amount of truncation and tailing, regardless of the nucleotide composition of the tail. Any spots on the same diagonal share the same final length and would be indistinguishable on an RNA gel blot, but based on this plot, would have different $3^{\prime}$ ends. We provide several examples in Figure 2. For example, Arabidopsis miR166 is significantly truncated and mildly tailed in the hen 1-8 mutant, with a small degree of truncation and tailing visible even in wild-type plants (Fig. 2, on left). In Drosophila and zebrafish, there is no obvious difference for miRNAs in a mutant hen 1 background, presumably because miRNAs are not methylated in animals; however, we were able to detect a small amount of $3^{\prime}$ variation in wild-type animals (Fig. 2, middle and right). This could reflect variation in processing or posttranscriptional $3^{\prime}$ modifications resulting from processes similar to those that we have described in plants.

\section{CONCLUSIONS}

The combination of these analysis and visualization tools will enable dissection of sRNA sequencing data to identify numerous attributes of the posttranscriptional modifications that occur to sRNAs. For example, it will be possible to compare the degree of truncation and tailing across miRNA families within a single organism to assess variability in $3^{\prime}$ modifications; these may reflect sequence-specific effects or other factors that have yet to be described. As an example, it has recently been published that AGO10 in Arabidopsis specifically binds miR166 and promotes the degradation of this miRNA (Ji et al. 2011; Zhu et al. 2011), so it will be interesting to apply these tools to identify $3^{\prime}$ modifications that may be promoted by AGO10. Given that hen 1 mutants are now available for rice, cross-species comparisons of $3^{\prime}$ modifications for orthologous miRNAs may also identify conserved or divergent decay destinies for specific miRNAs. One advantage of sequencing-based approaches for studying posttranscriptional modifications to sRNAs is that it is an open-ended analysis that can test

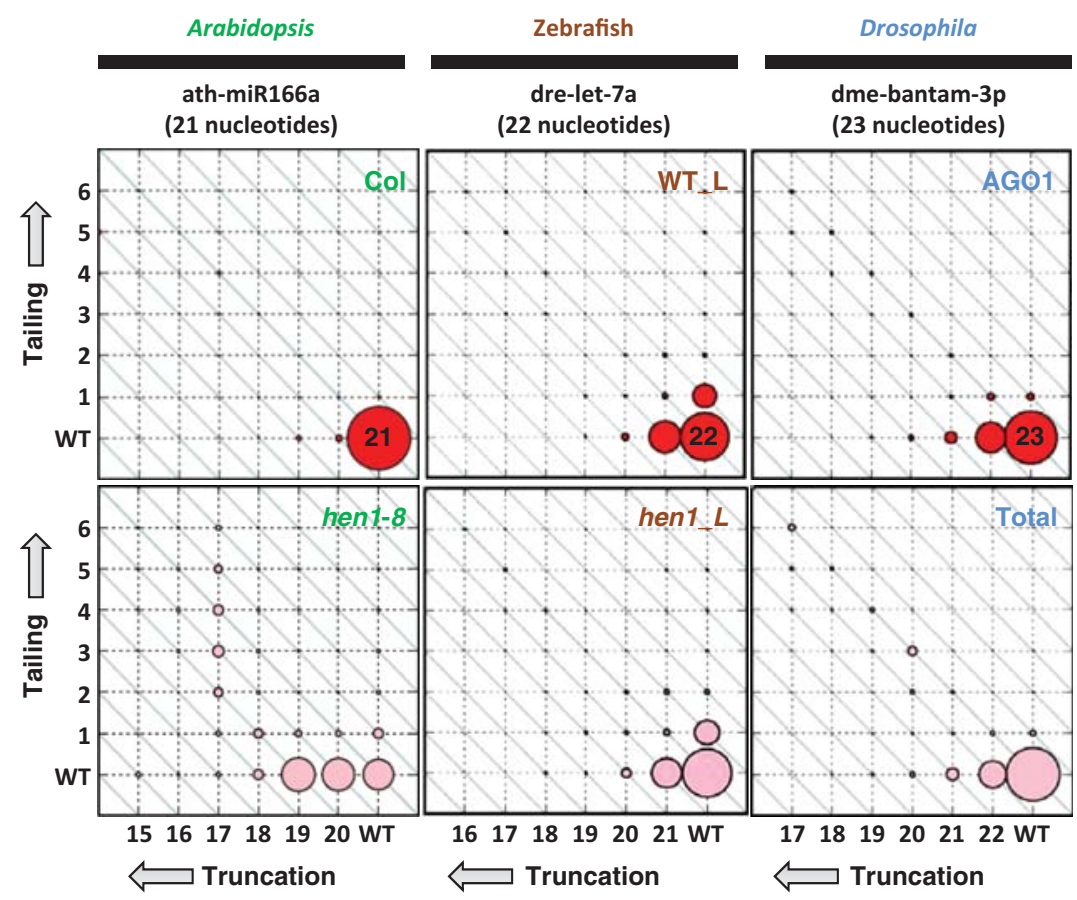

Figure 2. Matrix for summarizing and visualizing miRNA $3^{\prime}$ truncation and tailing from deep sequencing data. For each matrix, the $x$ axis represents the length of the $5^{\prime}$ genome-matched component (5GMC) of a particular miRNA related sequence; the $y$ axis represents the length of the "tail" added to the 5GMC, mostly through uridylation. Annotated miRNA sequences are considered to have no truncation or tail and therefore are positioned at wild type, wild type (WT, WT) in the lower right corner of each matrix. In this figure, we show the results for a single miRNA (named at the top) from two libraries in each of three organisms, as labeled. The canonical miRNA size is indicated in the spot at the lower right of the top row of panels. (Lower row) For Arabidopsis and zebrafish, we analyzed a hen 1 mutant, whereas in Drosophila, we compared AGO1-IPed sRNAs with total sRNAs. Different colored spots in the top and bottom sets of panels represent different libraries. The data are interpreted as described in the main text. The sRNA sequencing data used in this analysis are described in previous studies (see Table 1 for summary). The GEO accession numbers for the libraries that we analyzed are GSE35479 (Arabidopsis), GSE33582 (zebrafish), and GSE18806 (Drosophila). 
all known miRNAs in a single sequencing run. Furthermore, the data facilitate the analysis of other types of sRNAs, including heterochromatic siRNAs and transacting siRNAs, although alternative analysis approaches may be required to characterize the truncation and tails of such heterogeneous populations of sRNAs. We should also point out that there are a number of challenges or limitations to the approaches that we have described. These analyses depend on the accuracy of the miRNA annotation, and recent global analyses have shown that many miRBase-annotated miRNAs have siRNA-like qualities that may reflect older annotations that predate more strict, modern standards (Jeong et al. 2011). Biological factors can also confound the analyses, including (1) inconsistency in processing of a single precursor (optimal analyses require just one predominant miRNA species per precursor), (2) complex families with many loci that give rise to the same mature miRNAs, (3) weakly abundant miRNAs for which insufficient reads are available.

In conclusion, we believe that these are exciting times in which to study the posttranscriptional events that regulate sRNA function and stability. Numerous plant mutants have been described recently, led by the highly productive forward- and reverse-genetics approaches applied in the Chen laboratory that have identified a handful of genes critical to $3^{\prime}$ modifications of sRNAs. The biochemistry of these genes remains to be worked out, but deep sequencing and genome-wide approaches comprise a powerful and sensitive readout for the activity of these genes. Future rounds of analyses will likely identify determinants that distinguish the varied activity of these proteins on different sRNAs, whereas cross-species and even cross-kingdom comparative analyses will provide insights into the biology of these processes.

\section{ACKNOWLEDGMENTS}

This material is based on work supported by the National Institute of Food and Agriculture, United States Department of Agriculture, under Agreement no. 201267013-19396. We also gratefully acknowledge ongoing collaborations with Xuemei Chen and her laboratory members (University of California-Riverside) and Bing Yang and his laboratory members (Iowa State University). Manoj Pillay was helpful with earlier implementations of the algorithms that we describe.

\section{REFERENCES}

Abe M, Yoshikawa T, Nosaka M, Sakakibara H, Sato Y, Nagato Y, Itoh J. 2010. WAVY LEAF1, an ortholog of Arabidopsis HEN1, regulates shoot development by maintaining MicroRNA and trans-acting small interfering RNA accumulation in rice. Plant Physiol 154: 1335-1346.

Ameres SL, Horwich MD, Hung JH, Xu J, Ghildiyal M, Weng Z, Zamore PD. 2010. Target RNA-directed trimming and tailing of small silencing RNAs. Science 328: 1534-1539.

Bartel DP. 2004. MicroRNAs: Genomics, biogenesis, mechanism, and function. Cell 116: 281-297.

Baulcombe D. 2004. RNA silencing in plants. Nature 431: $356-363$.
Chapman EJ, Carrington JC. 2007. Specialization and evolution of endogenous small RNA pathways. Nat Rev Genet 8: 884-896.

Chen X. 2005. microRNA biogenesis and function in plants. FEBS Lett 579: 5923-5931.

Chen X, Liu J, Cheng Y, Jia D. 2002. HEN1 functions pleiotropically in Arabidopsis development and acts in C function in the flower. Development 129: 1085-1094.

Cuperus JT, Carbonell A, Fahlgren N, Garcia-Ruiz H, Burke RT, Takeda A, Sullivan CM, Gilbert SD, Montgomery TA, Carrington JC. 2010. Unique functionality of 22-nt miRNAs in triggering RDR6-dependent siRNA biogenesis from target transcripts in Arabidopsis. Nat Struct Mol Biol 17: 997-1003.

Deleris A, Gallego-Bartolome J, Bao J, Kasschau KD, Carrington JC, Voinnet O. 2006. Hierarchical action and inhibition of plant Dicer-like proteins in antiviral defense. Science 313: $68-71$.

Ghildiyal M, Xu J, Seitz H, Weng Z, Zamore PD. 2010. Sorting of Drosophila small silencing RNAs partitions microRNA* strands into the RNA interference pathway. RNA 16: 43-56.

Griffiths-Jones S, Saini HK, van Dongen S, Enright AJ. 2008. miRBase: Tools for microRNA genomics. Nucleic Acids Res 36: D154-D158.

Hamilton AJ, Baulcombe DC. 1999. A species of small antisense RNA in posttranscriptional gene silencing in plants. Science 286: 950-952.

Henderson IR, Zhang X, Lu C, Johnson L, Meyers BC, Green PJ, Jacobsen SE. 2006. Dissecting Arabidopsis thaliana DICER function in small RNA processing, gene silencing and DNA methylation patterning. Nat Genet 38: 721-725.

Horwich MD, Li C, Matranga C, Vagin V, Farley G, Wang P, Zamore PD. 2007. The Drosophila RNA methyltransferase, DmHen1, modifies germline piRNAs and single-stranded siRNAs in RISC. Curr Biol 17: 1265-1272.

Huang Y, Ji L, Huang Q, Vassylyev DG, Chen X, Ma JB. 2009. Structural insights into mechanisms of the small RNA methyltransferase HEN1. Nature 461: 823-827.

Ibrahim F, Rymarquis LA, Kim EJ, Becker J, Balassa E, Green PJ, Cerutti H. 2010. Uridylation of mature miRNAs and siRNAs by the MUT68 nucleotidyltransferase promotes their degradation in Chlamydomonas. Proc Natl Acad Sci 107: 3906-3911.

Iwasaki S, Kawamata T, Tomari Y. 2009. Drosophila argonaute1 and argonaute2 employ distinct mechanisms for translational repression. Mol Cell 34: 58-67.

Jeong DH, Park S, Zhai J, Gurazada SG, De Paoli E, Meyers BC, Green PJ. 2011. Massive analysis of rice small RNAs: Mechanistic implications of regulated microRNAs and variants for differential target RNA cleavage. Plant Cell 23: 4185-4207.

Ji L, Liu X, Yan J, Wang W, Yumul RE, Kim YJ, Dinh TT, Liu J, Cui X, Zheng B, et al. 2011. ARGONAUTE10 and ARGONAUTE1 regulate the termination of floral stem cells through two microRNAs in Arabidopsis. PLoS Genet 7: e1001358.

Jones-Rhoades MW, Bartel DP, Bartel B. 2006. MicroRNAS and their regulatory roles in plants. Annu Rev Plant Biol 57: 19-53.

Juliano C, Wang J, Lin H. 2011. Uniting germline and stem cells: The function of Piwi proteins and the piRNA pathway in diverse organisms. Annu Rev Genet 45: 447-469.

Kamminga LM, Luteijn MJ, den Broeder MJ, Redl S, Kaaij LJ, Roovers EF, Ladurner P, Berezikov E, Ketting RF. 2010. Hen 1 is required for oocyte development and piRNA stability in zebrafish. EMBO J 29: 3688-3700.

Kirino Y, Mourelatos Z. 2007a. The mouse homolog of HEN1 is a potential methylase for Piwi-interacting RNAs. RNA 13: 1397-1401.

Kirino Y, Mourelatos Z. 2007b. Mouse Piwi-interacting RNAs are 2'-O-methylated at their $3^{\prime}$ termini. Nat Struct Mol Biol 14: $347-348$.

Kurth HM, Mochizuki K. 2009. 2'-O-methylation stabilizes Piwi-associated small RNAs and ensures DNA elimination in Tetrahymena. RNA 15: 675-685. 
Lehrbach NJ, Armisen J, Lightfoot HL, Murfitt KJ, Bugaut A, Balasubramanian S, Miska EA. 2009. LIN-28 and the poly(U) polymerase PUP-2 regulate let-7 microRNA processing in Caenorhabditis elegans. Nat Struct Mol Biol 16: 1016-1020.

Li J, Yang Z, Yu B, Liu J, Chen X. 2005. Methylation protects miRNAs and siRNAs from a $3^{\prime}$-end uridylation activity in Arabidopsis. Curr Biol 15: 1501-1507.

Montgomery TA, Howell MD, Cuperus JT, Li D, Hansen JE, Alexander AL, Chapman EJ, Fahlgren N, Allen E, Carrington JC. 2008. Specificity of ARGONAUTE7-miR390 interaction and dual functionality in TAS3 trans-acting siRNA formation. Cell 133: 128-141.

Ohara T, Sakaguchi Y, Suzuki T, Ueda H, Miyauchi K. 2007. The $3^{\prime}$ termini of mouse Piwi-interacting RNAs are 2'-Omethylated. Nat Struct Mol Biol 14: 349-350

Ramachandran V, Chen X. 2008. Degradation of microRNAs by a family of exoribonucleases in Arabidopsis. Science 321: $1490-1492$

Reinhart BJ, Weinstein EG, Rhoades MW, Bartel B, Bartel DP 2002. MicroRNAs in plants. Genes Dev 16: 1616-1626.

Ren G, Chen X, Yu B. 2012. Uridylation of miRNAs by hen1 suppressor1 in Arabidopsis. Curr Biol 22: 695-700.

Rubio-Somoza I, Cuperus JT, Weigel D, Carrington JC. 2009. Regulation and functional specialization of small RNA-target nodes during plant development. Curr Opin Plant Biol 12: $622-627$.

Sunkar R, Chinnusamy V, Zhu J, Zhu JK. 2007. Small RNAs as big players in plant abiotic stress responses and nutrient deprivation. Trends Plant Sci 12: 301-309.

van Wolfswinkel JC, Claycomb JM, Batista PJ, Mello CC, Berezikov E, Ketting RF. 2009. CDE-1 affects chromosome segregation through uridylation of CSR-1-bound siRNAs. Cell 139: $135-148$
Voinnet O. 2009. Origin, biogenesis, and activity of plant microRNAs. Cell 136: 669-687.

Volpe T, Martienssen RA. 2011. RNA interference and heterochromatin assembly. Cold Spring Harb Perspect Biol 3: a003731.

Wierzbicki AT, Haag JR, Pikaard CS. 2008. Noncoding transcription by RNA polymerase Pol IVb/Pol V mediates transcriptional silencing of overlapping and adjacent genes. Cell 135: $635-648$.

Yang Z, Ebright YW, Yu B, Chen X. 2006. HEN1 recognizes 21-24 nt small RNA duplexes and deposits a methyl group onto the $2^{\prime} \mathrm{OH}$ of the $3^{\prime}$ terminal nucleotide. Nucleic Acids Res 34: 667-675.

Yu B, Yang Z, Li J, Minakhina S, Yang M, Padgett RW, Steward R, Chen X. 2005. Methylation as a crucial step in plant microRNA biogenesis. Science 307: 932-935.

Yu B, Bi L, Zhai J, Agarwal M, Li S, Wu Q, Ding SW, Meyers BC, Vaucheret H, Chen X. 2010. siRNAs compete with miRNAs for methylation by HEN1 in Arabidopsis. Nucleic Acids Res 38: 5844-5850.

Zhai J, Jeong DH, De Paoli E, Park S, Rosen BD, Li Y, Gonzalez AJ, Yan Z, Kitto SL, Grusak MA, et al. 2011. MicroRNAs as master regulators of the plant NB-LRR defense gene family via the production of phased, trans-acting siRNAs. Genes Dev 25: $2540-2553$.

Zhao Y, Yu Y, Zhai J, Ramachandran V, Dinh TT, Meyers BC, Mo B, Chen X. 2012. The Arabidopsis nucleotidyl transferase HESO1 uridylates unmethylated small RNAs to trigger their degradation. Curr Biol 22: 689-694.

Zhu H, Hu F, Wang R, Zhou X, Sze SH, Liou LW, Barefoot A, Dickman M, Zhang X. 2011. Arabidopsis Argonaute10 specifically sequesters miR166/165 to regulate shoot apical meristem development. Cell 145: 242-256. 


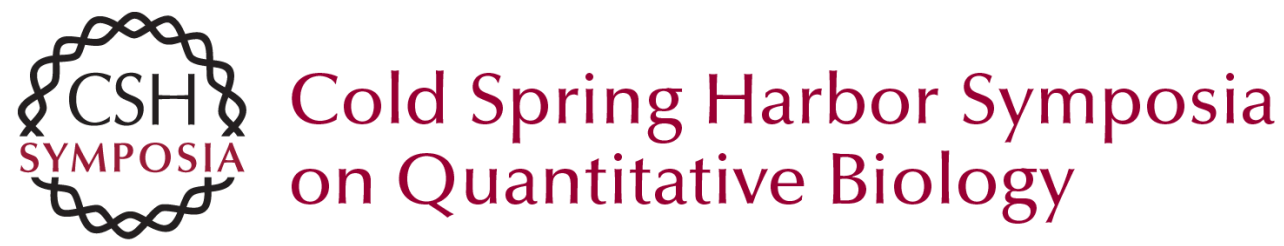

\section{Deep Sequencing from hen1 Mutants to Identify Small RNA 3 'Modifications}

J. Zhai and B.C. Meyers

Cold Spring Harb Symp Quant Biol 2012 77: 213-219 originally published online February 7, 2013

Access the most recent version at doi:10.1101/sqb.2013.77.014779

References This article cites 44 articles, 15 of which can be accessed free at: http://symposium.cshlp.org/content/77/213.full.html\#ref-list-1

\section{License}

Email Alerting Service the box at the top right corner of the article or click here. 